\title{
Is KELEA (kinetic energy limiting electrostatic attraction) a source of chemical energy?
}

\begin{abstract}
Chemical reactions involve the exchange of energy between interactive molecules, which result in the formation of different molecules. The first Law of Thermodynamics stipulates that chemical reactions cannot create new energy. It follows, therefore, that energy input is required in those chemical reactions in which the resulting molecules have more intrinsic energy than the original interactive molecules. It has been assumed that the formation of carbohydrates plus oxygen from relatively lower energy water and carbon dioxide molecules is solely achieved by the transformation of sunlight energy into chemical energy during photosynthesis. The chemical energy initially incorporated into carbohydrates is transferrable to subsequent energy-requiring chemical reactions. Sunlight energy is accordingly viewed as the originating energy in the chemical synthesis of all carbon-based molecules present within living organisms, some of which are subsequently consumed by other organisms as food. It is further assumed that oxidative phosphorylation of consumed food provides the sole source of cellular energy in animals and humans. Recent studies have identified an additional or alternative cellular energy (ACE) pathway in support of cellular activities. This pathway involves the absorption of an environmental force termed KELEA (kinetic energy limiting electrostatic attraction). This energy is expressed as an enhanced dynamic (kinetic) quality of the body's fluids. The present paper considers the possibility that KELEA may be associated with molecules and mediate most of the energy exchanges occurring in chemical reactions. Support for the notion that KELEA can be equated with chemical energy is provided by studies on the significantly increased energy output of KELEA activated gasoline, when compared with nonactivated gasoline.
\end{abstract}

Keywords: chemistry, alternative cellular energy, photosynthesis, oxidative phosphorylation, water, calories, enerceuticals ${ }^{\mathrm{TM}}$, electrical charge
Volume I Issue 2 - 2017

\author{
W John Martin \\ Institute of Progressive Medicine, USA
}

Correspondence: $W$ John Martin, Institute of Progressive Medicine, 1634 Spruce Street, South, Pasadena CA USA 91030 , Tel (626) 616-2868, Email wjohnmartin@ccid.org

Received: June 23, 2017 | Published: June 28, 2017

\begin{abstract}
Abbreviations: KELEA, kinetic energy limiting electrostatic attraction; ACE, alternative cellular energy; ADP, adenosine diphosphate; ATP, adenosine triphosphate; CPE, cytopathic effect; UV, ultraviolet; GC, ms gas chromatograph-mass spectroscopy; EDX, energy dispersive $\mathrm{x}$-ray
\end{abstract}

\section{Introduction}

All matter is composed of atoms, many of which are combined into molecules. Apart from water, which consists of an oxygen atom bound separately to two hydrogen atoms, the major compounds in living organisms are carbon containing molecules. These include lipid hydrocarbons (carbon plus hydrogen), carbohydrates (carbon plus hydrogen plus oxygen), proteins (carbon plus hydrogen plus oxygen plus nitrogen) and nucleic acids (carbon plus hydrogen plus oxygen plus nitrogen plus phosphorus). These and related groupings of compounds are collectively referred to as organic molecules. The remaining compounds in existence are termed inorganic molecules. Atoms can also remain unbound. The study of chemistry includes the process by which atoms can be switched between the bound and unbound states. It also includes the chemical reactions by which the atoms within a molecule or group of molecules can rearrange to yield different molecules.

Several types of chemical bonding occur between atoms and between molecules. i) Unbound atoms and atoms within molecules can be electrically charged due either to having one to three greater or lesser number of electrons, when compared with the number of protons. Atoms with more electrons than protons are electrically negative, while atoms with fewer electrons than protons are positively charged. Atoms with opposing charges can bind electro statically in ionic bonds. ii) Different atoms can bind closer to one another in a covalent manner, which involves the sharing (overlapping) of electrons. Atoms with a propensity to contribute shared electrons are termed nucleophiles, while those able to accept shared electrons are termed electrophiles. iii) A third type of atom binding is typical of metals and results from the external placing of pooled outer electrons from multiple atoms. The pooled electrons act as an enveloping electrostatic constraint on the movement of the enclosed atoms. iv) Molecules can also loosely interact electro statically if the charge distribution within individual atoms undergoes minor coordinated oscillations, or if they are dipolar with regions of excess positive and negative charges. One example is the rapid on-off binding that occurs between dipolar water molecules in the liquid state. The covalent bonding of oxygen with the hydrogen atoms involves sharing of the single electrons from the two hydrogen atoms with the electrons of the oxygen atom. This leaves each hydrogen atom with a slightly positive charge. Conversely, the oxygen atom acquires a slight negative charge. The opposing charges on the hydrogen and oxygen atoms of different water molecules lead to transient, yet repetitive intermolecular bonding. The resulting loose cohesion is referred to as hydrogen bonding and explains why water remains liquid between 0 and 100 degrees centigrade. ${ }^{1}$ Dipolar molecules can also self-assemble to form polymers and other aggregates. 


\section{Chemical energy}

All chemical reactions are based on the proposal that unbound atoms or molecules lose some "free" energy when they bind with one another. ${ }^{2}$ The energy loss is presumably directly proportional to the tightness of the inter-atom or inter-molecule bonding. The lost energy is regained upon separation of the atoms or molecules. Chemical reactions can proceed without a net source of external energy if the aggregate level of "free" energy in a set of molecules (reactants) is greater than the aggregate level of energy in a resulting new set of molecules (products). For some chemical reactions, the reduction in chemical energy is expressed as heat (exothermic reactions). In many complex chemical reactions, however, the reduction in chemical energy in one reaction provides a source of chemical energy for an interconnected or downstream chemical reaction, in which the products have higher energy than the reactants. An energy stimulus is often required to initiate chemical reactions. Typically, the triggering energy is regained as the chemical reaction proceeds. Enzymes are organic molecules, which facilitate interactions between specific reactants and, thereby, trigger (catalyze) chemical reactions. ${ }^{3}$

An overall net external energy source is required for chemical reactions in which the aggregate level of energy of the products exceeds that of the reactants. Net energy for chemical reactions can be provided by heat, light or, as already mentioned, by linkage to a chemical reaction that supplies the required chemical energy.

\section{Photosynthesis}

It was assumed that the chemical energy in organic molecules was originally derived from sunlight through the process of photosynthesis occurring in plants and in certain bacteria. ${ }^{4}$ Sunlight acting on chlorophyll molecules in plants results in the transfer of hydrogen ions to one side of a lipid membrane. The hydrogen ions return across the membrane through portals, which contain the enzyme ATP synthase. This enzyme catalyzes the addition of a third phosphate ion to adenosine diphosphate (ADP) to yield adenosine triphosphate (ATP). The generated ATP is used by the enzyme rubisco to convert water plus carbon dioxide into carbohydrate (glyceraldehyde) and oxygen. ${ }^{5}$ (Pairs of glyceraldehyde molecules are subsequently combined to form glucose.) Plants proceed to metabolize the glucose in an oxygen dependent process, termed oxidative phosphorylation, which occurs within mitochondria. In this complex process, glucose is reconverted to carbon dioxide and water in a manner that provides chemical energy to regenerate ATP from ADP. The ATP is then available to produce (synthesize) additional organic molecules in the plant, and to perform other energy-requiring cellular functions. Animals and humans consume plant generated glucose along with other synthesized organic molecules as food. ${ }^{6}$ The consumed food is metabolized (converted) to carbon dioxide, water, urea and other waste products. Food metabolism provides chemical energy to convert ADP to ATP, which then serves as a source of chemical energy for the animals and humans.

\section{Does photosynthesis fully equate with availa- ble bioenergy?}

A challenge with the above interpretation of life is its failure to explain the source of energy to account for the initial formation of lipid membranes able to partition hydrogen ions, and for the synthesis of highly complex molecules such as chlorophyll, ATP synthase, rubisco and the many enzymes and substrates involved in oxidative phosphorylation.
Another difficulty with presuming that life is solely driven by food metabolism is the limited amounts of chemical energy in foods. The energy in food is measured in terms of the heat generated during its oxygen dependent combustion to carbon dioxide and water. It is expressed as joules or Calories, with one Calorie being the energy required to raise the temperature of a kilogram of water by one degree centigrade. As reasoned elsewhere, a typical adult human expends more biological energy than provided by the Calories consumed in a typical daily diet. $^{5}$

A third reason to question the exclusive role of photosynthesis in providing all new chemical energy is the abiotic synthesis of organic molecules. Various organic molecules have been discovered on meteorites and other extraterrestrial bodies. ${ }^{6,7}$ An even more striking example is the abiotic synthesis of lipids mediated by the alternative cellular energy (ACE) pathway.

\section{ACE pathway}

The studies which led to the early description of the ACE pathway have been described in prior publications. ${ }^{8-14}$ Essentially, a cellular repair process was identified in cell cultures infected with stealth adapted viruses. The cellular repair process is attributed to the production by the infected cells of energy converting materials, which self-assemble into extracellular particles, fibers and threads. The cytopathic (cell damaging) effect (CPE) of the virus recurs shortly after the tissue culture medium containing the particles is replaced with fresh tissue culture medium. The reactivation can be prevented by adding a few particles to the re-feeding tissue culture medium. ${ }^{15}$ Similar intra- and extracellular particles are present in brain biopsies of stealth adapted virus infected patients and animals. ${ }^{16}$ A striking feature of the infected cells is the marked disruption of the cells' mitochondria, the major source of cellular energy. It was concluded that cellular survival was being maintained by the energy converting particles. Since many of the particles are pigmented, they are called alternative cellular energy (ACE) pigments. Gas chromatographMass spectroscopy (GC-MS) of ACE pigments reveals a wide range of small aromatic and aliphatic molecules, many being unidentifiable using established libraries of known compounds. Energy Dispersive X-ray (EDX) spectroscopy shows the incorporation of a range of various minerals, selectively distributed as limited combinations within different individual particles.

The energy related properties of ACE pigments include their being electrostatic and, therefore, dipolar and self-assembling; fluorescent under both ultraviolet (UV) and visible light, especially when interacting with certain dyes, including neutral red; kinetic in the sense of small particles moving in water far more than could be accountable by Brownian motion; and occasionally ferromagnetic. Vapor bubbles will commonly form about the larger particles when placed into water. Relevant to the present paper is the synthesis of abundant lipid-like materials in long-term maintained culture tubes containing ACE pigments but no remaining viable cells. The lipids being formed include fine particles, long narrow troughs, membranous sheets, solid crystals, pyramids and more complex pigmented collections, which progressively increase in size. A photograph of complex lipid material, which formed many months after all viable cells had disintegrated from a stealth adapted virus culture tube is shown in (Figure 1). The lipids have only been partially characterized by thin layer chromatography and the additional presence of terpenes has not been excluded. Abiotic lipid synthesis also occurs in tissue culture medium and on agar plates in which ACE pigment particles 
are placed. As published previously, ${ }^{17}$ lipid production also occurs with ACE pigment particles present in dried perspiration of stealth adapted virus infected patients (Figure 2).

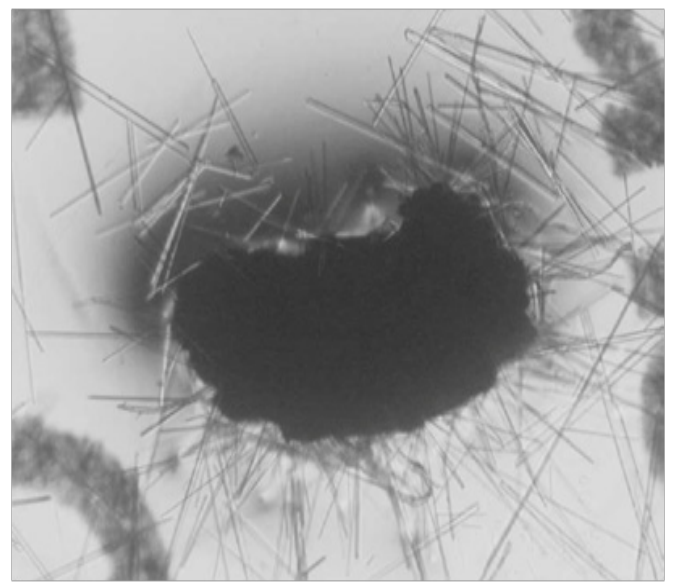

Figure I Collections of yellow-orangedeposits in a culture tube in which stealth adapted viruses had been cultured. All viable cells had long since disintegrated from the culture, although it did contain residual ACE pigments. The collections formed over several months and are still apparent in tubes maintained for over 15 years. Dark-field microscopy. Magnification $\times 200$.

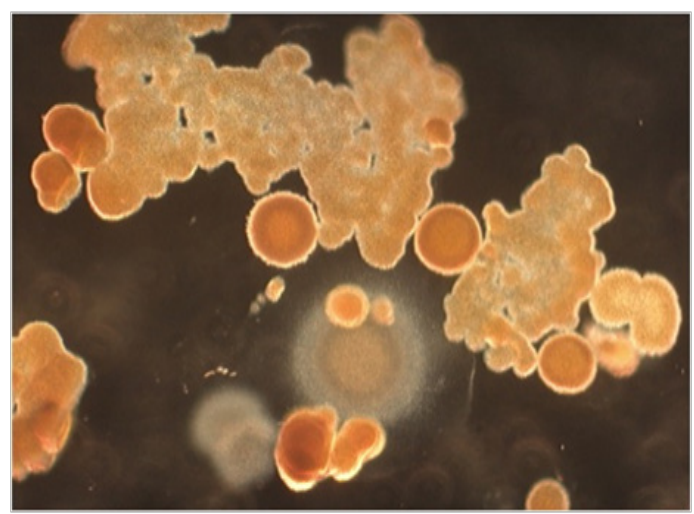

Figure 2 Formation of numerous needle shaped crystals around a patientderived ACE pigment particle placed on an agar plate. The needles formed over several days. Dark-field microscopy. Magnification $\times 200$.

\section{KELEA (Kinetic energy limiting electrostatic attraction)}

ACE pigments are considered to function as an antenna for the attraction of a natural force termed KELEA (kinetic energy limiting electrostatic attraction). ${ }^{18-26}$ The fundamental role of KELEA is presumed to be the prevention of fusion of electro statically attracted opposite electrical charges. It appears that KELEA is attracted to electrical charges, including the separated electrical charges on dipolar molecules. Certain dipolar molecules can release KELEA to nearby water, possibly in an oscillatory manner. The effective transmission of KELEA to water reduces the strength of the inter-molecular bonding between the water molecules and may also directly contribute to the movements (kinetic activity) of the water molecules. There are multiple ways in which water and other fluids can become activated with KELEA. Some of these methods involve the addition to the fluids of dipolar molecules, also called enerceuticals ${ }^{\mathrm{TM}}$. These molecules can subsequently be removed by decanting, filtration or progressive dilutions, as in homeopathy. Another approach is to place fluids near heightened KELEA energy fields, as established with various energy based devices, typically with rapid on-off electrical switching. These approaches are reviewed elsewhere. ${ }^{18-26}$

\section{KELEA as a potential expression of chemical energy}

The nature of the free energy, which diminishes as molecules bind with one another, is not clearly defined in chemistry textbooks. Nor is the mechanism whereby chemical reactions are interconnected such that allows for energy to be transferred. Given that most chemical reactions are based on electrical attraction, it is reasonable to assume that KELEA is involved. Separated opposing electrical charges possess a potential electrical energy, which correlates directly with the voltages of the charges and inversely with the square of the distance between the charges. Potential electrical energy can convert into kinetic energy as the opposing charges approach one another. A similar phenomenon might be expected of the levels of KELEA associated with separated molecules.

Regarding energy transfer, one suggestion is that water comprises micro domains. ${ }^{27}$ The water molecules in these domains are either loosely bonded (low-density) or more tightly bonded (high-density) bonded water molecules. The water in the low-density domains is proposed as having more energy than the water in the high-density domains. The energy producing phase of a chemical reaction could lead to the formation of low-density water domains from high-density water domains. Reconversion of the low-density water domain back to a high-density domain could then be a source of energy for the remaining phase of the chemical reaction. This interesting hypothesis still does not explain abiotic synthesis, nor the mechanism whereby molecules seemingly regain previously lost energy upon the breaking of chemical bonds.

The postulate that KELEA is equivalent to some form of chemical energy is attractive since it can explain both issues. The KELEA postulate is clearly applicable to reactants in which there are opposing electrical charges. It is known, however, that some reactive molecules have an equal number of protons and electrons and are, therefore, electrically neutral. Even so, the protons and electrons in the reacting molecules would be recognizable by KELEA.

\section{Rubisco and other oscillating chemical reac- tions}

Rubisco is the basic enzyme of photosynthesis and is reportedly the most abundant protein in nature. It has an exceptionally low reaction rate of processing only 5 molecules of carbon dioxide per second. The reaction with carbon dioxide also shows a $20 \%$ inhibition by oxygen molecules. ${ }^{28}$ By these criteria, it is very inefficient. At limiting reagent concentrations, as expected in plants, rubisco has a markedly fluctuating activity. Arguably, other naturally fluctuating biochemical reactions may be operating as an antenna to attract KELEA and transfer it into chemical energy.

\section{Supporting evidence from the combustion of KELEA activated gasoline}

Various ways have been described to transfer KELEA into water. Activation can be measured as increased kinetic activity, reduced surface tension, increased volatility and other methods. KELEA can also activate other fluids, including ethanol and gasoline. Even 
simple methods of gasoline activation can achieve greater than $10 \%$ increased mileage. ${ }^{29}$ While this are minor reductions in the low percentages of carbon monoxide and unburnt hydrocarbons formed, the increased mileage is clearly consistent with KELEA activated gasoline acquiring added chemical energy.

\section{Conclusion}

This paper outlines a novel concept that an energy force termed KELEA (kinetic energy limiting electrostatic attraction) is fundamentally involved in chemical reactions by providing an exchangeable energy between molecules. KELEA is attracted to the electrical charges within atoms and molecules. A proportion of the attached energy is released as the molecules bind with one another. Conversely, bound molecules can undergo loosening or even dissociate from each other if provided with additional KELEA. Water can play a major role in transferring KELEA between the molecules involved in chemical reactions.

Reversing the membrane partitioning of opposing electrical charges through ion channels may have initiated the primordial synthesis of organic molecules, leading to more complex processes, such as photosynthesis and oxidative phosphorylation. Rubisco is the primary enzyme responsible for photosynthesis. In addition to this activity, it may play a more basic role as an antenna for KELEA, as may also other naturally oscillating chemical reactions.

The finding of significantly increased mileage from KELEA activated gasoline is consistent with the postulated equating KELEA with chemical energy.

\section{Acknowledgements}

The Institute of Progressive Medicine is a component of MI Hope Inc., a non-profit public charity.

\section{Conflict of interest}

The author declares no conflict of interest.

\section{References}

1. Ludwig R. Water: From Hydrogen Bonding to Dynamics and Structure. Germany: Wiley-VCH Verlag GmbH; 2011. 400 p.

2. Lotti S, Raff L, Sabatini A. Chemical and biochemical thermodynamics. Is it time for a reunification? Biophysical Chemistry. 2017;221:49-57.

3. Robinson PK. Enzymes: principles and biotechnological applications Essays Biochem. 2015;59:1-41.

4. Pessarakli M. Handbook of Photosynthesis. 3rd ed. Florida: CRC Press; 2016. $846 \mathrm{p}$.

5. Anderson I, Backlund A. Structure and Function of Rubisco. Plant Physiol Biochem. 2008;46(3):275-291.

6. Coultate T. Food. The Chemistry of its Components. 6th ed. UK: Royal Society Chemistry; 2015. 599 p.

7. Martin WJ. Hyper-excitability followed by functional quiescence in neuronal cells caused by insufficient cellular energy (ICE):Treatable by enhancing the alternative cellular energy (ACE) pathway. World Journal Neuroscience (in press). 2017;7(3):257-266.

8. Pizzarello S, Cooper GW, Flynn GJ. The Nature and Distribution of the Organic Material in Carbonaceous Chondrites and Interplanetary Dust Particles. In Meteorites and the Early Solar System II. Univ Arizona Press; 2006. p. 625-651.
9. De Sanctis MC, Ammannito E, McSween HY, et al. Localized aliphatic organic material on the surface of Ceres. Science. 2017;355(6326):719722

10. Martin WJ, Zeng LC, Ahmed K, et al. Cytomegalovirus-related sequences in an atypical cytopathic virus repeatedly isolated from a patient with the chronic fatigue syndrome. Am J Pathol. 1994;145(2):440-451.

11. Martin WJ, Ahmed KN, Zeng LC, et al. African green monkey origin of the atypical cytopathic 'stealth virus' isolated from a patient with chronic fatigue syndrome. Clin DiagVirol. 1995;4(1):93-103.

12. Martin WJ. Stealth virus isolated from an autistic child. J Autism Dev Dis. 1995;25:223-224.

13. Martin WJ, Glass RT. Acute encephalopathy induced in cats with a stealth virus isolated from a patient with chronic fatigue syndrome. Pathobiology. 1995;63(3):115-118.

14. Martin WJ. Severe stealth virus encephalopathy following chronicfatigue-syndrome-like illness: Clinical and histopathological features. Pathobiology. 1996;64(1):1-8.

15. Martin WJ. Stealth virus culture pigments: A potential source of cellular energy. Exp Mol Path. 2003;74(3):210-223.

16. Martin WJ. Complex intracellular inclusions in the brain of a child with a stealth virus encephalopathy. Exp Mol Path. 2003;74(3):179-209.

17. Martin WJ. Alternative cellular energy pigments mistaken for parasitic skin infestations. Exp Mol Pathol. 2005;78(3):212-214.

18. Martin WJ. KELEA Activated Water-Enhancing the Alternative Cellular Energy (ACE) pathway. In Stealth Adapted Viruses; Alternative Cellular Energy (ACE) \& KELEA Activated Water. Author House IN; 2014. p. 115-144.

19. Martin WJ (2014) The alternative cellular energy (ACE) pathway in the repair of the Cytopathic effect (CPE) Caused by Stealth Adapted Viruses: In Vitro and in Vivo Evidence Supporting a New Therapeutic Paradigm. In Stealth Adapted Viruses; Alternative Cellular Energy $(A C E) \&$ KELEA Activated Water. Author House, Bloomington IN, 2014. p. 31-70.

20. Martin WJ. KELEA: A Natural Energy That Seemingly Reduces Intermolecular Hydrogen Bonding In Water and Other Liquids. Open Journal of Biophysics. 2015;5:69-79.

21. Martin W. KELEA activation of water and other fluids for health, agriculture and industry. Journal of Water Resource and Protection. 2015; 7:1331-1344.

22. Martin WJ. Interactive electric fields attract KELEA (kinetic energy limiting electrostatic attraction) and can lead to the activation of water. Int Journal Complementary \& Alternative Med. 2015;1(6):00034.

23. Martin WJ. Interacting light paths attract KELEA (kinetic energy limiting electrostatic attraction) and can lead to the activation of water. Open J Biophysics. 2015;5(4):115-121.

24. Martin WJ, Laurent D. Homeopathy as a Misnomer for Activation of the Alternative Cellular Energy Pathway: Evidence for the therapeutic benefits of Enercel in a diverse range of clinical illnesses. Int $J$ Complement Alt Med. 2015;2(1):00045.

25. Martin WJ. Alternative cellular energy. A unifying concept in complementary alternative medicine. Int Journal Complementary \& Alternative Medicine. 2015;1(4):00022.

26. Martin WJ. Insufficiency of cellular energy (ICE): The basis for many illnesses potentially correctable using KELEA activated water. Int $J$ Complement Alt Med. 2016;4(1):00106.

27. Wiggins P. Life Depends upon Two Types of Water. PLoS ONE. 2008;3(1):e1406. 
28. Spreitzer RJ, Salvucci ME. Rubisco: Structure, Regulatory Interactions, and Possibilities for a Better Enzyme. Annu Rev Plant Biol. 2002;53:449-475.
29. Martin WJ. KELEA (Kinetic Energy Limiting Electrostatic Attraction) Can Markedly Improve the Performance of Gasoline and Diesel Fuels in Power Generation and in Transportation. Journal of Transportation Technologies. 2016;6(3):148-154. 